\title{
Nonlinear Electrodynamics: Alternative Field Theory for Featuring Photon Propagation Over Weak Background Electromagnetic Fields and what Earth Receivers Read off Radio Signals from Interplanetary Spacecraft Transponders
}

\author{
Herman J. Mosquera Cuesta ${ }^{1,2,3}$ \\ ${ }^{1}$ Departmento de Física Universidade Estadual Vale do Acaraú \\ Avenida da Universidade 850, Campus da Betânia, CEP 62.040-370, Sobral, Ceará \\ ${ }^{2}$ Instituto de Cosmologia, Relatividade e Astrofísica (ICRA-BR) \\ Centro Brasileiro de Pesquisas Físicas, Rua Dr. Xavier Sigaud 150, \\ CEP 22290-180, Urca Rio de Janeiro, RJ \\ ${ }^{3}$ International Center for Relativistic Astrophysics Network (ICRANet) \\ International Coordinating Center, Piazzalle della Repubblica 10, 065112, Pescara \\ 1,2 Brazil \\ ${ }^{3}$ Italy
}

\section{Introduction}

A few observational and/or experimental results have dramatically pushed forward the research program on gravity as those from the radio-metric Doppler tracking received from the Pioneer 10 and 11 spacecrafts when the space vehicles were at heliocentric distances between 20 and 70 Astronomical Units (AU). These data have conclusively demonstrated the presence of an anomalous, tiny and blue-shifted frequency drift that changes smoothly at a rate of $\sim 6 \times 10^{-9} \mathrm{~Hz} \mathrm{~s}^{-1}$. Those signals, if interpreted as a gravitational pull of the Sun on each Pioneer vehicle, translates into a deceleration of $a_{P}=(8.74 \pm 1.33) \times 10^{-10} \mathrm{~m} \mathrm{~s}^{-2}$. This sunward acceleration appears to be a violation of Newton's inverse-square law of gravitation, and is referred to as the Pioneer anomaly, the nature of which remains still elusive to unveil. Within the theoretical framework of nonlinear electrodynamics (NLED) in what follows we will address this astrodynamical puzzle, which over the last fifteen years has challenged in a fundamental basis our understanding of gravitational physics. To this goal we will first, and briefly, review the history of the Pioneers 10 and 11 missions. Then a synopsis of currently available Lagrangian formulations of NLED is given. And finally, we present our solution of this enigma by invoking a special class of NLED theories featuring a proper description of electromagnetic phenomena taking place in environments where the strength of the (electro)magnetic fields in the background is decidedly low. 


\section{What is the problem: The Pioneer anomaly}

In this short voyage to the Pioneer 10 and 11 missions our main guide will be the comprehensive and richly documented recent review on the Pioneer Anomaly by [Turyshev, S. G. \& Toth, V. T. (2010). Living Rev. Rel. 13 (2010) 4. arXiv:1001.3686, v2, gr-qc] from which we retake some ideas and references. (The attentive readers are kinldy addressed to this invaluable article).

The Pioneer 10 and 11 spacecrafts were the first two man-made space vehicles designed to explore the outer solar system. The trajectories of the spaceships were projected to passage nearby Jupiter during 1972-1973 having as objectives to conduct exploratory investigation of the interplanetary medium beyond the orbit of Mars, the nature of the asteroid belt, the environmental and atmospheric characteristics of Jupiter and Saturn (for Pioneer 11), and to investigate the solar system beyond the orbit of the Jovian planet. ${ }^{1}$

The Pioneer missions were the first space probes to adventure over the asteroid belt, heading for close-up observations of the gaseous giant planets, and for performing in situ studies of the physical properties of the interplanetary medium in the outer solar system. The design of their missions was guided by the simplicity, having a powerful rocket-launching system to push the spacecrafts on an hyperbolic trajectory aimed directly at Jupiter, which the spacecrafts were expected to fly-by approximately 21 months after launch (see Fig. 1).

By the late 1960's, the aerospace engineering technology available to the designers of the Pioneer missions made it no longer practical to use solar panels for operating a spacecraft at large distances, as for instance that of Jupiter. A cause of this, a built-in nuclear power system, in the form of radioisotope thermoelectric generators (RTGs) powered by ${ }^{238} \mathrm{Pu}$, was chosen as the means to provide electrical power to the spaceship. As even this was relatively new technology at the time the missions were designed, the power subsystem was suitably over-engineered, being the unique design requirement to have a completely functional space probe capable of performing all planned scientific tasks by running only three (out of four) RTGs.

The entire design of these spacecrafts and their science missions was characterized by such conservative engineering, and for sure it was responsible for both the exceptional longevity of the two spacecrafts and their ability to deliver science results which by far exceeded the expectations of their designers.

The original plan envisioned a primary mission of two to three years in duration. Nevertheless, following its encounter with Jupiter, Pioneer 10 remained functional for over 30 years. Meanwhile, Pioneer 11, though not as long lived as its engineering-copy craft, successfully navigated a path across the solar system for another encounter with Saturn, offering the first close-up observations of the ringed planet. After the encounters with Jupiter and Saturn (for Pioneer 11, see Fig. 1), the space ships followed, near the plane of the ecliptic, hyperbolic orbits of escape heading to opposite sides of the solar system, continuing their extended missions. The spacecrafts explored the outer regions of the solar system, studying energetic particles from the Sun (solar wind), and cosmic rays entering our neighborhood in the Milky Way. (Their cousin spacecrafts, the Voyager1 and 2, that where launched contemporarily, studied in the beginning of their mission, the interplanetary space, what resulted in a very accurate mapping of the interplanetary magnetic field and its strength, as one can see in Fig. 2 below).

\footnotetext{
${ }^{1}$ See details on the Pioneer missions at http://www.nasa.gov/centers/ames/missions/ archive/pioneer.html. Be awared that another member of Pioneer spacecrafts family, Pioneer 6, remained operational for more than 35 years after launch.
} 


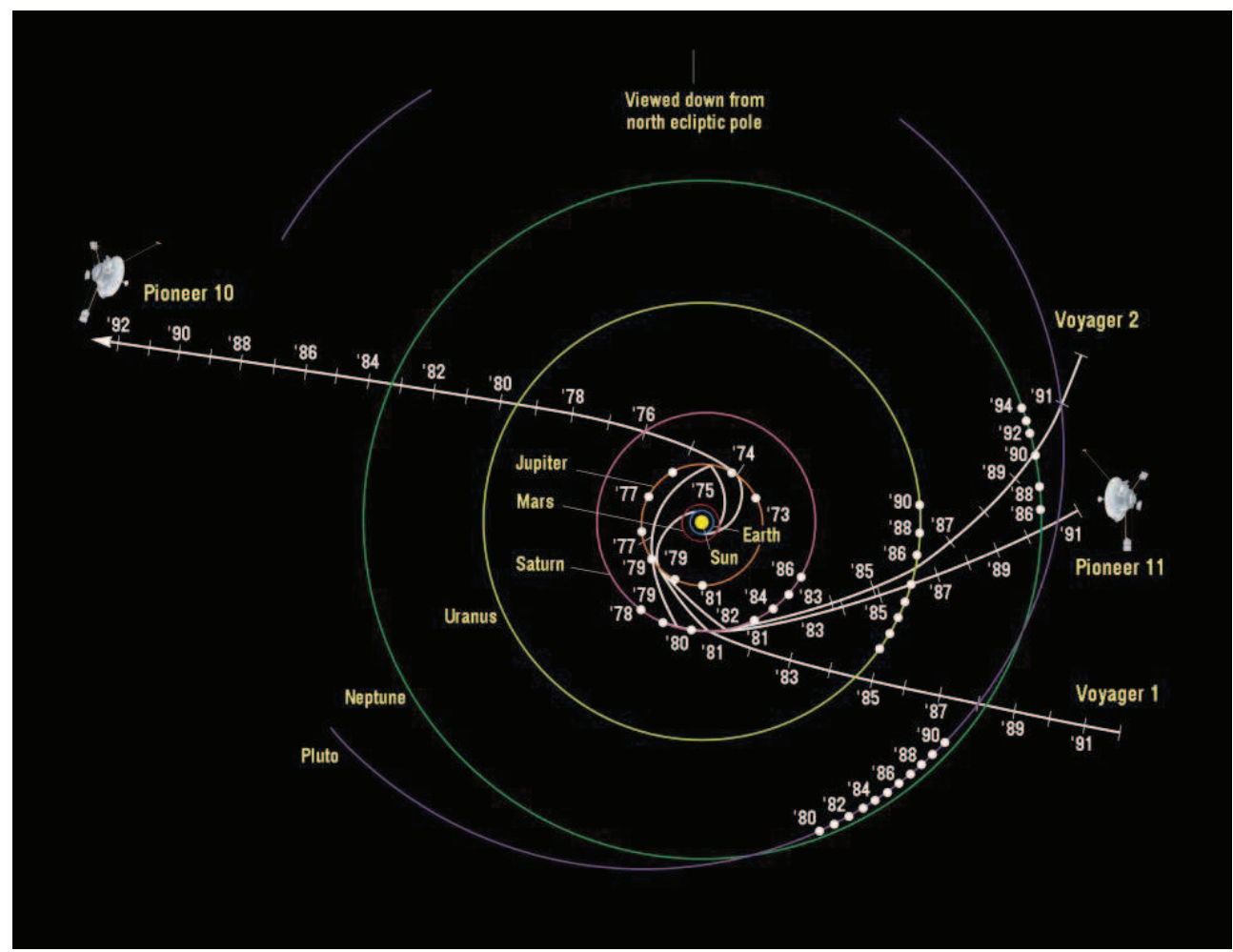

Fig. 1. Ecliptic pole view of the spacecrafts Pioneer 10 and 11 interplanetary trajectories (see also the trajectories of the vehicles Voyager 1 and 2). Credit:

http://www.nasa.gov/centers/ames/missions/archive/pioneer.html

In virtue of a combination of many factors, the Pioneers were excellent space sondes for pursuing experiments of high precision celestial mechanics. This includes the presence of a coherent mode transceiver on board, the attitude control (spin-stabilized, with a minimum number of attitude correction maneuvers using thrusters), the design of the power system (the RTGs being on extended booms aided the stability of the craft and also helped in reducing thermal effects), and Doppler tracking of high precision (with the accuracy of post-fit Doppler residuals at the level of $\mathrm{mHz}$ ). The exceptional built-in sensitivity to acceleration of the Pioneer 10 and 11 spacecrafts naturally allowed them to reach a level of accuracy of $\sim 10^{-10}$ $\mathrm{m} / \mathrm{s}^{2}$. The result was one of the most precise spacecraft navigations in deep space since the early days of space exploration. That is the great legacy of the Pioneer missions.

After having had a brief accounting of the Pioneers missions, one can proceed to review our current understanding of nonlinear electrodynamics and to settle down the foundations for its use in the search for a solution to the Pioneer anomaly. In this Section we shall briefly review the theoretical foundations of some theories of NLED, focusing essentially on the fundamental prediction concerning the way photons propagate through a vacuum space permeated by electromagnetic (EM) fields: The fact that photons travel along the effective metric, and not over the geometry in the background. It is this peculiar feature what makes the photon to 
"feel" itself being acted upon by a force, and consequently to undergo acceleration. ${ }^{2}$ In our understanding, such effect is responsible for the drift in frequency undergone by the photon. Next we will show that any NLED, independently of the specific form of its Lagrangian, brings in such a frequency shift. And in our view, it is such acceleration what can account for the Pioneer anomaly.

\section{Some Lagrangian formulations of nonlinear electrodynamics}

To start with, it is worth to recall that according to quantum electrodynamics (QED: see Delphenich $(2003$; 2006) for a complete review on NLED and QED) a vacuum has nonlinear properties (Heisenberg \& Euler 1936; Schwinger 1951) which affect the photon propagation. A noticeable advance in the realization of this theoretical prediction has been provided by [Burke, Field, Horton-Smith , etal., 1997), who demonstrated experimentally that the inelastic scattering of laser photons by gamma-rays in a background magnetic ield is definitely a nonlinear phenomenon. The propagation of photons in NLED has been examined by several authors [Bialynicka-Birula \& Bialynicki-Birula, 1970; Garcia \& Plebanski, 1989; Dittrich \& Gies, 1998; De Lorenci, Klippert, Novello, etal., 2000; Denisov, Denisova \& Svertilov, 2001a, 2001b, Denisov \& Svertilov, 2003]. In the geometric optics approximation, it was shown by [Novello, De Lorenci, Salim \& etal., 2000; Novello \& Salim, 2001], that when the photon propagation is identified with the propagation of discontinuities of the EM field in a nonlinear regime, a remarkable feature appears: The discontinuities propagate along null geodesics of an effective geometry which depends on the EM field on the background. This means that the NLED interaction can be geometrized. An immediate consequence of this NLED property is the prediction of the phenomenon dubbed as photon acceleration, which is nothing else than a shift in the frequency of any photon traveling over background electromagnetic fields. The consequences of this formalism are examined next.

\subsection{Heisenberg-Euler approach}

The Heisenberg-Euler Lagrangian for nonlinear electrodynamics (up to order 2 in the truncated infinite series of terms involving F) has the form Heisenberg \& Euler (1936)

$$
L_{\mathrm{H}-\mathrm{E}}=-\frac{1}{4} F+\bar{\alpha} F^{2}+\bar{\beta} G^{2},
$$

where $F=F_{\mu \nu} F^{\mu \nu}$, with $F_{\mu \nu}=\partial_{\mu} A_{\nu}-\partial_{\nu} A_{\mu}$, and $G=\frac{1}{2} \eta_{\alpha \beta \gamma \delta} F^{\alpha \beta} F^{\gamma \delta}=-4 \vec{E} \cdot \vec{B}$, with greek index running $(0,1,2,3)$, while $\bar{\alpha}$ and $\bar{\beta}$ are arbitrary constants.

When this Lagrangian is used to describe the photon dynamics the equations for the EM field in vacuum coincide in their form with the equations for a continuum medium in which the electric permittivity and magnetic permeability tensors $\epsilon_{\alpha \beta}$ and $\mu_{\alpha \beta}$ are functions of the electric and magnetic fields determined by some observer represented by its 4-vector velocity $V^{\mu}$ [Denisov, Denisova \& Svertilov, 2001a, 2001b; Denisov \& Svertilov, 2003; Mosquera Cuesta \& Salim, 2004a, 2004b]. The attentive reader must notice that this first order approximation is valid only for $B$-fields smaller than $B_{q}=\frac{m^{2} c^{3}}{c \bar{h}}=4.41 \times 10^{13} \mathrm{G}$ (Schwinger's critical $B$-field Schwinger (1951)). In curved spacetime, these equations are written as

\footnotetext{
${ }^{2}$ Because of the special theory of relativity constraints regarding the propagation of any perturbation, it becomes clear that such effect must manifest itself as a change in one or both of their physical properties: its frequency or its wavelength. Hence, through the Pioneer spacecrafts radio Doppler tracking we might be observing the effect on the photon frequency.
} 


$$
\begin{gathered}
D_{\| \alpha}^{\alpha}=0, \quad B_{\| \alpha}^{\alpha}=0, \\
D_{\| \beta}^{\alpha} \frac{V^{\beta}}{c}+\eta^{\alpha \beta \rho \sigma} V_{\rho} H_{\sigma \| \beta}=0, \\
B_{\| \beta}^{\alpha} \frac{V^{\beta}}{c}-\eta^{\alpha \beta \rho \sigma} V_{\rho} E_{\sigma \| \beta}=0 .
\end{gathered}
$$

Here, the vertical bars subscript " $\|$ " stands for covariant derivative and $\eta^{\alpha \beta \rho \sigma}$ is the antisymmetric Levi-Civita tensor.

The 4-vectors representing the electric and magnetic fields are defined as usual in terms of the electric and magnetic fields tensor $F_{\mu \nu}$ and polarization tensor $P_{\mu \nu}$

$$
\begin{aligned}
E_{\mu} & =F_{\mu \nu} \frac{V^{v}}{c}, & B_{\mu} & =F_{\mu \nu}^{*} \frac{V^{v}}{c}, \\
D_{\mu} & =P_{\mu \nu} \frac{V^{v}}{c}, & H_{\mu} & =P_{\mu \nu}^{*} \frac{V^{v}}{c},
\end{aligned}
$$

where the dual tensor $X_{\mu \nu}^{*}$ is defined as $X_{\mu \nu}^{*}=\frac{1}{2} \eta_{\mu v \alpha \beta} X^{\alpha \beta}$, for any antisymmetric second-order tensor $X_{\alpha \beta}$.

The meaning of the vectors $D^{\mu}$ and $H^{\mu}$ comes from the Lagrangian of the EM field, and in the vacuum case they are given by

$$
H_{\mu}=\mu_{\mu \nu} B^{v}, \quad D_{\mu}=\epsilon_{\mu v} E^{v},
$$

where the permeability and tensors are given as

$$
\begin{aligned}
& \mu_{\mu v}=\left[1+\frac{2 \alpha}{45 \pi B_{q}^{2}}\left(B^{2}-E^{2}\right)\right] h_{\mu \nu}-\frac{7 \alpha}{45 \pi B_{q}^{2}} E_{\mu} E_{v}, \\
& \epsilon_{\mu v}=\left[1+\frac{2 \alpha}{45 \pi B_{q}^{2}}\left(B^{2}-E^{2}\right)\right] h_{\mu v}+\frac{7 \alpha}{45 \pi B_{q}^{2}} B_{\mu} B_{v} .
\end{aligned}
$$

In these expressions $\alpha$ is the EM coupling constant $\left(\alpha=\frac{e^{2}}{\hbar c}=\frac{1}{137}\right)$. The tensor $h_{\mu v}$ is the metric induced in the reference frame perpendicular to the observers determined by the vector field $V^{\mu}$.

Meanwhile, as we are assuming that $E^{\alpha}=0$, then one gets

$$
\epsilon_{\beta}^{\alpha}=\epsilon h_{\beta}^{\alpha}+\frac{7 \alpha}{45 \pi B_{q}^{2}} B^{\alpha} B_{\beta}
$$

and $\mu_{\alpha \beta}=\mu h_{\alpha \beta}$. The scalars $\epsilon$ and $\mu$ can be read directly from Eqs. $(8,9)$ as

$$
\epsilon \equiv \mu=1+\frac{2 \alpha}{45 \pi B_{q}^{2}} B^{2} .
$$

Applying conditions (62) and (63) (derived in the Appendix) to the field equations when $E^{\alpha}=$ 0 , we obtain the constraints $e^{\mu} \epsilon_{\mu v} k^{v}=0$ and $b^{\mu} k_{\mu}=0$ and the following equations for the discontinuity fields $e_{\alpha}$ and $b_{\alpha}$ : 


$$
\begin{gathered}
\epsilon^{\lambda \gamma} e_{\gamma} k_{\alpha} \frac{V^{\alpha}}{c}+\eta^{\lambda \mu \rho \nu} \frac{V_{\rho}}{c}\left(\mu b_{v} k_{\mu}-\mu^{\prime} \lambda_{\alpha} B_{v} k_{\mu}\right)=0, \\
b^{\lambda} k_{\alpha} \frac{V^{\alpha}}{c}-\eta^{\lambda \mu \rho \nu} \frac{V_{\rho}}{c}\left(e_{v} k_{\mu}\right)=0 .
\end{gathered}
$$

Isolating the discontinuity field from (12), substituting in equation (13), and expressing the products of the completely anti-symmetric tensors $\eta_{\nu \xi \gamma \beta} \eta^{\lambda \alpha \rho \mu}$ in terms of delta functions Stephani (2004), we obtain

$$
\begin{aligned}
& b^{\lambda}\left(k_{\alpha} k^{\alpha}\right)^{2}+\left(\frac{\mu^{\prime}}{\mu} l_{\beta} b^{\beta} k_{\alpha} B^{\alpha}+\frac{\beta B_{\beta} b^{\beta} B_{\alpha} k^{\alpha}}{\mu-\beta B^{2}}\right) k^{\lambda}+ \\
& \left(\frac{\mu^{\prime}}{\mu l_{\alpha} b^{\alpha}}\left(k_{\beta} V^{\beta}\right)^{2}\left(k_{\alpha} k^{\alpha}\right)^{2}-\frac{\beta B_{\alpha} b^{\alpha}\left(k_{\beta} k^{\beta}\right)^{2}}{\mu-\beta B^{2}}\right) B^{\lambda}-\left(\frac{\mu^{\prime}}{\mu} l_{\mu} b^{\mu} k_{\alpha} B^{\alpha} k_{\beta} V^{\beta}\right) V^{\lambda}=0 .
\end{aligned}
$$

This expression is already squared in $k_{\mu}$ but still has an unknown $b_{\alpha}$ term. To get rid of it, one multiplies by $B_{\lambda}$, to take advantage of the EM wave polarization dependence. By noting that if $B^{\alpha} b_{\alpha}=0$ one obtains the dispersion relation by separating out the $k^{\mu} k^{v}$ term, what remains is the (-) effective metric. Similarly, if $B_{\alpha} b^{\alpha} \neq 0$, one simply divides by $B_{\gamma} b^{\gamma}$ so that by factoring out $k^{\mu} k^{\nu}$, what results is the (+) effective metric. For the case $B_{\alpha} b^{\alpha}=0$, one obtains the standard dispersion relation

$$
g^{\alpha \beta} k_{\alpha} k_{\beta}=0 .
$$

whereas for the case $B_{\alpha} b^{\alpha} \neq 0$, the result is

$$
\left[\left(1+\frac{\mu^{\prime} B}{\mu}+\frac{\tilde{\beta} B^{2}}{\mu-\tilde{\beta} B^{2}}\right) g^{\alpha \beta}-\frac{\mu^{\prime} B}{\mu} \frac{V^{\alpha} V^{\beta}}{c^{2}}+\left(\frac{\mu^{\prime} B}{\mu}+\frac{\tilde{\beta} B^{2}}{\mu-\tilde{\beta} B^{2}}\right) l^{\alpha} l^{\beta}\right] k_{\alpha} k_{\beta}=0,
$$

where $(')$ stands for $\frac{d}{d B}$, and we have defined

$$
\tilde{\beta}=\frac{7 \alpha}{45 \pi B_{q}^{2}}, \quad \text { and } \quad l^{\mu} \equiv \frac{B^{\mu}}{\left|B^{\gamma} B_{\gamma}\right|^{1 / 2}}
$$

as the unit 4-vector along the $B$-field direction.

From the above expressions we can read the effective metric $g_{+}^{\alpha \beta}$ and $g_{-}^{\alpha \beta}$, where the labels "+" and "-" refers to extraordinary and ordinary polarized rays, respectively. Then, we need the covariant form of the metric tensor, which is obtained from the expression defining the inverse metric $g_{\mu v} g^{v \alpha}=\delta_{\mu}^{\alpha}$. So that one gets from one side

and from the other

$$
g_{\mu \nu}^{-}=g_{\mu \nu}
$$

$$
\begin{array}{r}
g_{\mu \nu}^{+}=\left(1+\frac{\mu^{\prime} B}{\mu}+\frac{\beta B^{2}}{\mu-\beta B^{2}}\right)^{-1} g_{\mu v} \\
+\left[\frac{\mu^{\prime} B}{\mu\left(1+\frac{\mu^{\prime} B}{\mu}+\frac{\beta B^{2}}{\mu-\beta B^{2}}\right)\left(1+\frac{\beta B^{2}}{\mu-\beta B^{2}}\right)}\right] \frac{V_{\mu} V_{v}}{c^{2}}+\left(\frac{\frac{\mu^{\prime} B}{\mu}+\frac{\beta B^{2}}{\mu-\beta B^{2}}}{1+\frac{\mu^{\prime} B}{\mu}+\frac{\beta B^{2}}{\mu-\beta B^{2}}}\right) l_{\mu} l_{v} .
\end{array}
$$


The function $\frac{\mu^{\prime} B}{\mu}$ can be expressed in terms of the magnetic permeability of the vacuum, and is given as

$$
\frac{\mu^{\prime} B}{\mu}=2\left(1-\frac{1}{\mu}\right)
$$

Thus equation (19) indicates that the photon propagates on an effective metric.

\subsection{Born-Infeld theory}

The propagation of light can also be viewed within the framework of the Born-Infeld Lagrangian. Such theory is inspired in the special theory of relativity, and indeed it incorporates the principle of relativity in its construction, since the fact that nothing can travel faster than light in a vacuum is used as a guide to establishing the existence of an upper limit for the strength of electric fields around an isolated charge, an electron for instance. Such charge is then forced to have a characteristic size Born \& Infeld (1934). The Lagrangian then reads

$$
L=-\frac{b^{2}}{2}\left[\left(1+\frac{F}{b^{2}}\right)^{1 / 2}-1\right] .
$$

As in this particular case, the this Lagrangian is a functional of the invariant $F$, i.e., $L=L(F)$, but not of the invariant $G \equiv B_{\mu} E^{\mu}$, the study of the NLED effects turns out to be simpler (here again we suppose $E=0$ ). In the equation above, $b=\frac{e}{R_{0}^{2}}=\frac{e}{\frac{e^{4}}{m_{0}^{2} c^{8}}}=\frac{m_{0}^{2} c^{8}}{e^{3}}=9.8 \times 10^{15}$ e.s.u.

In order to derive the effective metric that can be deduced from the B-I Lagrangian, one has therefore to work out, as suggested in the Appendix, the derivatives of the Lagrangian with respect to the invariant $F$. The first and second derivatives then reads

$$
L_{\mathrm{F}}=\frac{-1}{4\left(1+\frac{F}{b^{2}}\right)^{1 / 2}} \quad \text { and } \quad L_{\mathrm{FF}}=\frac{1}{8 b^{2}\left(1+\frac{F}{b^{2}}\right)^{3 / 2}} .
$$

The $L(F)$ B-I Lagrangian produces, according to Eq.(70) in the Appendix, an effective contravariant metric given as

$$
g_{\text {eff }}^{\mu v}=\frac{-1}{4\left(1+\frac{F}{b^{2}}\right)^{1 / 2}} g^{\mu v}+\frac{B^{2}}{2 b^{2}\left(1+\frac{F}{b^{2}}\right)^{3 / 2}}\left[h^{\mu v}+l^{\mu} l^{v}\right] .
$$

Both the tensor $h_{\mu v}$ and the vector $l^{\mu}$ in this equation were defined earlier (see Eqs.(9) and (16) above).

Because the geodesic equation of the discontinuity (that defines the effective metric, see the Appendix) is conformal invariant, one can multiply this last equation by the conformal factor $-4\left(1+\frac{F}{b^{2}}\right)^{3 / 2}$ to obtain

$$
g_{\text {eff }}^{\mu v}=\left(1+\frac{F}{b^{2}}\right) g^{\mu \nu}-\frac{2 B^{2}}{b^{2}}\left[h^{\mu \nu}+l^{\mu} l^{\nu}\right] .
$$

Then, by noting that

$$
F=F_{\mu v} F^{\mu v}=-2\left(E^{2}-B^{2}\right),
$$


and recalling our assumption $E=0$, then one obtains $F=2 B^{2}$. Therefore, the effective metric reads

$$
g_{\text {eff }}^{\mu v}=\left(1+\frac{2 B^{2}}{b^{2}}\right) g^{\mu v}-\frac{2 B^{2}}{b^{2}}\left[h^{\mu v}+l^{\mu} l^{\nu}\right],
$$

or equivalently

$$
g_{\text {eff }}^{\mu v}=g^{\mu \nu}+\frac{2 B^{2}}{b^{2}} V^{\mu} V^{v}-\frac{2 B^{2}}{b^{2}} l^{\mu} l^{\nu}
$$

As one can check, this effective metric is a functional of the background metric $g^{\mu v}$, the 4-vector velocity field of the inertial observers $V^{v}$, and the spatial configuration (orientation $\left.l^{\mu}\right)$ and strength of the $B$-field.

Thus the covariant form of the background metric can be obtained by computing the inverse of the effective metric $g_{\text {eff }}^{\mu v}$ just derived. With the definition of the inverse metric $g_{\text {eff }}^{\mu v} g_{v \alpha}^{\text {eff }}=\delta^{\mu}{ }_{\alpha}$, the covariant form of the effective metric then reads

$$
g_{\mu \nu}^{\text {eff }}=g_{\mu \nu}-\frac{2 B^{2} / b^{2}}{\left(2 B^{2} / b^{2}+1\right)} V_{\mu} V_{v}+\frac{2 B^{2} / b^{2}}{\left(2 B^{2} / b^{2}+1\right)} l_{\mu} l_{v},
$$

which is the result that we were looking for. The terms additional to the background metric $g_{\mu \nu}$ characterize any effective metric.

\subsection{Pagels-Tomboulis Abelian theory}

In 1978, the Pagels-Tomboulis nonlinear Lagrangian for electrodynamics appeared as an effective model of an Abelian theory introduced to describe a perturbative gluodynamics model. It was intended to investigate the non trivial aspects of quantum-chromodynamics (QCD ) like the asymptotic freedom and quark confinement Pagels \& Tomboulis (1978). In fact, Pagels and Tomboulis argued that:

"since in asymptotically free Yang-Mills theories the quantum ground state is not controlled by perturbation theory, there is no a priori reason to believe that individual orbits corresponding to minima of the classical action dominate the Euclidean functional integral. "

In view of this drawback, of the at the time understanding of ground states in quantum theory, they decided to examine and classify the vacua of the quantum gauge theory. To this goal, they introduced an effective action in which the gauge field coupling constant $g$ is replaced by the effective coupling $\bar{g}(t) \cdot T=\ln \left[\frac{F_{\mu \nu}^{a} F^{a \mu v}}{\mu^{4}}\right]$. The vacua of this model correspond to paramagnetism and perfect paramagnetism, for which the gauge field is $F_{\mu \nu}^{a}=0$, and ferromagnetism, for which $F_{\mu \nu}^{a} F^{a \mu \nu}=\lambda^{2}$, which implies the occurrence of spontaneous magnetization of the vacuum. ${ }^{3}$ They also found no evidence for instanton solutions to the quantum effective action. They solved the equations for a point classical source of color spin, which indicates that in the limit of spontaneous magnetization the infrared energy of the field becomes linearly divergent. This leads to bag formation, and to an electric Meissner effect confining the bag contents.

This effective model for the low energy $(3+1)$ QCD reduces, in the Abelian sector, to a nonlinear theory of electrodynamics whose density Lagrangian $L(X, Y)$ is a functional of the invariants $X=F_{\mu v} F^{\mu v}$ and their dual $Y=\left(F_{\mu \nu} F^{\mu v}\right)^{\star}$, having their equations of motion given by

${ }^{3}$ This is the imprint that such theory describes nonlinear electrodynamics. 


$$
\nabla_{\mu}\left(-L_{X} F^{\mu v}-L_{Y}^{*} F^{\mu v}\right)=0,
$$

where $L_{X}=\partial L / \partial X$ and $L_{Y}=\partial L / \partial Y$. This equation is supplemented by the Faraday equation, i. e., the electromagnetic field tensor cyclic identity (which remains unchanged)

$$
\nabla_{\mu} F_{\nu \lambda}+\nabla_{\nu} F_{\lambda \mu}+\nabla_{\lambda} F_{\mu \nu}=0
$$

In the case of a simple dependence on $X$, the equations of motion turn out to be Kunze (2008) (here we put $C=0$ and $4 \gamma=-\left(\Lambda^{8}\right)^{(\delta-1) / 2}$ in the original Lagrangian given in Pagels \& Tomboulis (1978))

$$
L_{\delta}=-\frac{1}{4}\left(\frac{X^{2}}{\Lambda^{8}}\right)^{(\delta-1) / 2} X,
$$

where $\delta$ is an dimensionless parameter and $[\Lambda]=($ anenergyscale $)$. The value $\delta=1$ yields the standard Maxwell electrodynamics.

The energy-momentum tensor for this Lagrangian $L(X)$ can be computed by following the standard recipe, which then gives

$$
T_{\mu v}=\frac{1}{4 \pi}\left(L_{X} g^{a b} F_{\mu a} F_{b v}+g_{\mu \nu} L\right)
$$

while its trace turns out to be

$$
T=-\frac{1-\delta}{\pi}\left(\frac{X^{2}}{\Lambda^{8}}\right)^{(\delta-1) / 2} X .
$$

It can be shown Kunze (2008) that the positivity of the $T_{0}^{0} \equiv \rho$ component implies that $\delta \geq 1 / 2$. The Lagrangian (31) has been studied by Kunze (2008) for explaining the amplification of the primordial magnetic field in the Universe, being the analysis focused on three different regimes: 1) $B^{2} \gg E^{2}$, 2) $B^{2} \simeq \mathcal{O}\left(E^{2}\right)$, 3) $E^{2} \ll B^{2}$. It has also been used by Mosquera Cuesta \& Lambiase (2009) to discuss both the origin of the baryon asymmetry in the universe and the origin of primordial magnetic fields. More recently it has also been discussed in the review on " Primordial magneto-genesis" by Kandus (2010).

Because the equation of motion (29) above, exhibits similar mathematical aspect as eq. (35) (reproduced in the Section), it appears clear that the Pagels and Tomboulis Lagrangian (31) leads also to an effective metric identical to that one given in equation (40), below.

\subsection{Novello-Pérez Bergliaffa-Salim NLED}

More recently, Novello et al. (2004) (NPS) revisited the several general properties of nonlinear electrodynamics by assuming that the action for the electromagnetic field is that of Maxwell with an extra term, namely 4

$$
S=\int \sqrt{-g}\left(-\frac{F}{4}+\frac{\gamma}{F}\right) d^{4} x
$$

where $F \equiv F_{\mu \nu} F^{\mu v}$.

\footnotetext{
${ }^{4}$ Notice that this Lagrangian is gauge invariant, and that hence charge conservation is guaranteed in this theory.
} 
Physical motivations for bringing in this theory have been provided in Novello et al. (2004). Besides of those arguments, an equally unavoidable motivation comes from the introduction in the 1920's of both the Heisenberg-Euler and Born-Infeld nonlinear electrodynamics discussed above, which are valid in the regime of extremely high magnetic field strengths, i.e. near the Schwinger's limit. Both theories have been extensively investigated in the literature (see for instance Mosquera Cuesta \& Salim (2004a;b); Mosquera Cuesta et al. (2006) and the long list of references therein). Since in nature non only such very strong magnetic fields exist, then it appears to be promising to investigate also those super weak field frontiers. From the conceptual point of view, this phenomenological action has the advantage that it involves only the electromagnetic field, and does not invoke entities that have not been observed (like scalar fields) and/or speculative ideas (like higher-dimensions and brane worlds).

At first, one notices that for high values of the field $F$, the dynamics resembles Maxwell's one except for small corrections associate to the parameter $\gamma$, while at low strengths of $F$ it is the $1 / F$ term that dominates. (Clearly, this term should dramatically affect, for instance, the photon- $\vec{B}$ field interaction in intergalactic space, which is relevant to understand the solution to the Pioneer anomaly using NLED.). The consistency of this theory with observations, including the recovery of the well-stablished Coulomb law, was shown in Novello et al. (2004) using the cosmic microwave radiation bound, and also after discussing the anomaly in the dynamics of Pioneer 10 spacecraft Mbelek et al. (2007). Both analysis provide small enough values for the coupling constant $\gamma$ Mosquera Cuesta (2010).

\subsubsection{Photon dynamics in NPS NLED: Effective geometry}

Next we investigate the effects of nonlinearities in the evolution of EM waves in the vacuum permeated by background $\vec{B}$-fields. An EM wave is described onwards as the surface of discontinuity of the EM field. Extremizing the Lagrangian $L(F)$, with $F\left(A_{\mu}\right)$, with respect to the potentials $A_{\mu}$ yields the following field equation Plebanski (1970)

$$
\nabla_{v}\left(L_{F} F^{\mu v}\right)=0,
$$

where $\nabla_{v}$ defines the covariant derivative. Besides this, we have the EM field cyclic identity

$$
\nabla_{\nu} F^{* \mu v}=0 \quad \Leftrightarrow \quad F_{\mu v \mid \alpha}+F_{\alpha \mu \mid v}+F_{v \alpha \mid \mu}=0 .
$$

Taking the discontinuities of the field Eq.(35) one gets (all the definitions introduced here are given in Hadamard (1903)) ${ }^{5}$

$$
L_{F} f_{\lambda}^{\mu} k^{\lambda}+2 L_{F F} F^{\alpha \beta} f_{\alpha \beta} F^{\mu \lambda} k_{\lambda}=0,
$$

which together with the discontinuity of the Bianchi identity yields

$$
f_{\alpha \beta} k_{\gamma}+f_{\gamma \alpha} k_{\beta}+f_{\beta \gamma} k_{\alpha}=0 .
$$

A scalar relation can be obtained if we contract this equation with $k^{\gamma} F^{\alpha \beta}$, which yields

\footnotetext{
${ }^{5}$ Following Hadamard's method Hadamard (1903), the surface of discontinuity of the EM field is denoted by $\Sigma$. The field is continuous when crossing $\Sigma$, while its first derivative presents a finite discontinuity. These properties are specified as follows: $\left[F_{\mu v}\right]_{\Sigma}=0, \quad\left[F_{\mu v \mid \lambda}\right]_{\Sigma}=f_{\mu \nu} k_{\lambda}$, where the symbol $\left[F_{\mu \nu}\right]_{\Sigma}=\lim _{\delta \rightarrow 0^{+}}\left(\left.J\right|_{\Sigma+\delta}-\left.J\right|_{\Sigma-\delta}\right)$ represents the discontinuity of the arbitrary function $J$ through the surface $\Sigma$. The tensor $f_{\mu \nu}$ is called the discontinuity of the field, $k_{\lambda}=\partial_{\lambda} \Sigma$ is the propagation vector, and the symbols "|" and "|" stand for partial and covariant derivatives.
} 


$$
\left(F^{\alpha \beta} f_{\alpha \beta} g^{\mu \nu}+2 F^{\mu \lambda} f_{\lambda}^{v}\right) k_{\mu} k_{v}=0 .
$$

It is straightforward to see that here we find two distinct solutions: a) when $F^{\alpha \beta} f_{\alpha \beta}=0$, case in which such mode propagates along standard null geodesics, and b) when $F^{\alpha \beta} f_{\alpha \beta}=\chi$. In the case a) it is important to notice that in the absence of charge currents, this discontinuity describe the propagation of the wave front as determined by the field equation (35), above. Thence, following Lichnerowicz. (1962) the quantity $f^{\alpha \beta}$ can be decomposed in terms of the propagation vector $k_{\alpha}$ and a space-like vector $a_{\beta}$ (orthogonal to $k_{\alpha}$ ) that describes the wave polarization. Thus, only the light-ray having polarization and direction of propagation such that $F^{\alpha \beta} k_{\alpha} a_{\beta}=0$ will follow geodesics in $g_{\mu v}$. Any other light-ray will propagate on the effective metric (40). Meanwhile, in this last case, we obtain from equations (37) and (39) the propagation equation for the field discontinuities being given by Novello et al. (2000)

$$
\underbrace{\left(g^{\mu \nu}-4 \frac{L_{F F}}{L_{F}} F^{\mu \alpha} F_{\alpha}{ }^{v}\right)}_{\text {effective metric }} k_{\mu} k_{v}=0 .
$$

This equation proves that photons propagate following a geodesic that is not that one on the background space-time, $g^{\mu \nu}$, but rather they follow the effective metric given by Eq.(40), which depends on the background field $F^{\mu \alpha}$, i. e., on the $\vec{B}$-field.

\section{Understanding the Pioneer anomaly within NLED}

\subsection{Astrodynamics of Pioneer 10 and 11: Input facts}

As pointed out above, since 1998 the JPL group have continuously reported an anomalous frequency shift derived from about ten years study of radio-metric data from Pioneer 10: 03/01/1987-22/07/1998 Anderson et al. (1998), Pioneer 11: 05/01/1987-01/10/1990 Anderson et al. (1995). The group has also found a similar feature in the data from of Ulysses and Galileo spacecrafts Anderson et al. (1998; 2002). The observed effect mimics a constant sunward acceleration acting on the spacecraft with magnitude

$$
a_{P}=(8.74 \pm 1.33) \times 10^{-8} \mathrm{~cm} \mathrm{~s}^{-2}
$$

and a steady frequency $(v)$ drift

$$
\frac{d \Delta v}{d t} \simeq 6 \times 10^{-9} \mathrm{~Hz} / \mathrm{s}
$$

which equates to a "clock acceleration":

$$
\frac{d \Delta v}{d t}=\frac{a_{P}}{c} v,
$$

where $c$ represents tha speed of light in a vacuum, and $t$ is the one way signal travel time. An independent analysis of the radio-metric Doppler tracking data from the Pioneer 10 spacecraft for the period 1987 - 1994 confirms the previous observations Markwardt (2002). In addition, by removing the spin-rate change contribution yields an apparent anomalous acceleration $a_{P}=(7.84 \pm 0.01) \times 10^{-8} \mathrm{~cm} \mathrm{~s}^{-2}$, of the same amount for both Pioneer 10/11 Anderson et al. (2002); Abramovici \& Vager (1986). Besides, it has been noted that the magnitude of $a_{P}$ compares nicely to $\mathrm{cH}_{0}$, where $\mathrm{H}_{0}$ is the Hubble parameter today. 
As stressed above, unlike other spacecrafts like the Voyagers and Cassini which are three-axis stabilized (hence, not well-suited for a precise reconstitution of trajectory because of numerous attitude controls), the Pioneer 10/11, Ulysses and the by-now destroyed Galileo are attitude-stabilized by spinning about an axis (parallel to the axis of the high-gain antenna) which permits precise acceleration estimations to the level of $10^{-8} \mathrm{~cm} \mathrm{~s}^{-2}$ (single measurement accuracy averaged over 5 days). Besides, because of the proximity of Ulysses and Galileo to the Sun, the data from both spacecrafts were strongly correlated to the solar radiation pressure unlike the data from the remote Pioneer 10/11. Let us point out that the motions of the four spacecrafts are modelled by general relativistic equations (see Anderson et al. (2002), section $I V$ ) including the perturbations from heavenly bodies as small as the large main-belt asteroids (the Sun, the Moon and the nine planets are treated as point masses). Nonetheless, the observed frequency shift remains unexplained Turyshev \& Toth (2010).

Thenceforth, several proposals for dedicated missions to test the Pioneer anomaly are now under consideration Pioneer Collaboration (2005), in virtue of the dramatic implications of the Pioneer puzzle for the understanding of gravity.

\subsection{What has been done by other researchers}

In search for a possible origin of the anomalous blueshift, a number of gravitational and non-gravitational potential causes have been ruled out by Anderson et al. (2002). According to the authors, none of these effects may explain $a_{P}$ and some are 3 orders of magnitude or more too small. The addition of a Yukawa force to the Newtonian law does not work ease. An additional acceleration is predicted by taking into account the Solar quadrupole moment Mbelek \& Michalski (2002). Although this entails a blueshift, it decreases like the inverse of the power four of the heliocentric radius, being of the order of $a_{P}$ only below $2.1 \mathrm{AU}$.

Meanwhile, the claim that the Modified Newtonian Dynamics (MOND) may explain $a_{P}$ in the strongly Newtonian limit of MOND Quevedo (2005); Milgron (2001; 2002) is not obvious at all. First, the fits to the rotational curves of spiral galaxies yield for the MOND acceleration constant $a_{0}$ a value eight times smaller than $\mathrm{cH}_{0}$ Quevedo (2005). Second, the gravitational pulling of the Sun up to $100 \mathrm{AU}$ still yields an acceleration greater than $a_{0}$ by at least three orders of magnitude, equating $a_{0}$ only at about 3000 AU. Hence, Newtonian dynamics up to general relativity corrections should apply to the spacecrafts. Otherwise, one would be inclined to conclude that MOND is ruled out by a laboratory experiment Milgron $(2001 ; 2002)$. Now, any true Doppler shift would involve an accompanying acceleration, which would be in conflict with both the motions of planets and long-period comets Anderson et al. (1995); Iorio (2006a;b;c).

Heretofore what we have learnt is that based on Einstein-Maxwell equations, the only other photon frequency shift that can be misinterpreted, at the solar system scale, with the Doppler shift is the gravitational frequency shift. In the weak field and low velocity limit, this would involve a time dependent gravitational potential instead of a spatial dependent one. Such proposals invoking the dark energy as the source of the time dependent gravitational potential have been suggested Iorio (2006a;b;c); Tangen (2006). However, quintessence, like other fundamental scalar fields, has not yet been observed.

In summary, prosaic explanations, non-gravitational forces and modified dynamics or new interaction (long or short range) force terms do not work Mbelek \& Michalski (2002); Quevedo (2005); Milgron (2001; 2002); Rañada (2003; 2005). Gravitational origin of the anomaly is rouled out by the precision of the planetary ephemeris (see Anderson et al. (1998), Iorio (2006a;b;c), 
and others Tangen (2006)) and the known bounds on dark matter within the orbital radius of Uranus or Neptune Rañada (2003; 2005); Whitmire \& Matese (2003).

\subsection{What we are proposing to tackle the Pioneer anomaly}

By gathering together all the arguments reviewed above, one is led to the conclusion that the Pioneer anomaly does not seem to be related to the gravitational interaction Anderson et al. (1998); Iorio (2006a;b;c); Tangen (2006). If this is the case, what other of the currently known interactions in nature could afford a consistent understanding for the radio-metric Doppler tracking data from Pioneer spacecrafts?

The right answer could be related to the fact that there are only two long range interactions known today: Gravity and electromagnetism. Therefore, what remains is the EM sector. ${ }^{6}$ Meanwhile, the possibility of an interaction of the EM signal with the solar wind leading to a change of the frequency of the EM signal is now rouled out (see Anderson et al. (2002)).

Indeed, it appears to be unescapable to conclude that what we are observing (measuring through the receivers) could be related to the equation of motion of the photon. In other words, the mounting evidence seems to converge to what could be happening to the photon during its propagation through the interplanetary space from the Pioneer 10/11 antennas to the receivers on Earth.

It is timely, then, to recall that classical (Maxwell theory) or quantized (QED) linear electrodynamics does not allow for a change of the frequency of a photon during its propagation in a linear medium without invoking diffusion due to the interaction with the surrounding matter (hence a smear out of the image of the source). Moreover, for such a phenomenon to occur, one needs to consider a general and non trivial Lagrangian density $L=L(F)$ for which its second derivative w.r.t. $F: d^{2} L / d F^{2}=L_{F F} \neq 0$. Therefore, the Pioneer anomaly, if not an artifact, may be a result of NLED as we show below. Indeed, relation (43) above translates, in covariant notation, into

$$
\frac{d x^{v}}{d l} \nabla_{v} k^{\mu}=\frac{a_{P}}{c^{2}} k^{\mu}
$$

where $l$ is some affine parameter along a ray defined by $k^{\mu}=\frac{d x^{\mu}}{d l}$ (see Fujii \& Sasaki (2006)). The latter equation departs from the classical electrodynamics one (see Landau \& Lifchiftz (1970), section 87)

$$
\frac{d x^{v}}{d l} \nabla_{v} k^{\mu}=0
$$

and suggests the occurrence of the NLED effect dubbed photon acceleration.

The concept of photon acceleration, which follows from the description of photon propagation in NLED, was discussed by Novello \& Salim (2001), see also the book by Mendonça et al. (2006). Next we explain why the anomaly shows up in some situations and not others. (For experimental tests of NLED and further theoretical predictions see Mosquera Cuesta et al. (2006); Burke et al. (1997); Lundstrom et al. (2006); Lundin et al. (2006); Marklund \& Shukla (2006)).

Therefore, the alternative that the Pioneer anomaly is not consequence of an actual change in the spacecraft velocity (see Anderson et al. (2002), Section X) deserves to be investigated. Indeed, a direct interpretation of the observational data from the spacecrafts implies merely an anomalous time-dependent blueshift of the photons of the communication signals. On the

\footnotetext{
${ }^{6}$ Non-metric fields can also be regarded as gravitational fields and there is a lot of space for speculation.
} 
other hand, in using a time dependent potential Iorio (2006a;b;c); Tangen (2006) to explain the Pioneer 10/11 data one may be pointing out to the need of an effective metric for the photons. In fact, what is needed is just a time variation of the 4-momentum of the photon along its path. Thus the atomic energy levels would not be affected. Rather, only the motion of the photon being concerned.

\subsection{NLED at all distance scales: From cosmology down to astrodynamics in the Solar System}

Upon the collection of arguments presented above, it appears that all these requirements are achieved by considering that NLED is based on a Lagrangian density $L(F)$ which includes terms depending nonlinearly on the invariant $F=F_{\mu \nu} F^{\mu v}$, with $F=2\left(B^{2} c^{2}-E^{2}\right)$ Novello et al. (2000); Novello \& Salim (2001); Plebanski (1970), instead of the usual Lagrangian density $L=$ $-\frac{1}{4} F$ of classical electromagnetism in a vacuum. As stated above, we shall explore the effects of nonlinearities in the evolution of EM waves, which are envisioned onwards as the surface of discontinuity of the EM field. Therefore, as shown above, by extremizing the Lagrangian with respect to the potentials $A_{\mu}$ one obtains the EM field equation of motion Plebanski (1970) ${ }^{7}$

$$
\nabla_{v}\left(L_{F} F^{\mu v}\right)=0
$$

in which $\nabla_{v}$ represents the covariant derivative, and $L_{F}=d L / d F$.

Recalling the discussion above, the dynamics of the photon propagation follows the equation

$$
\left(g^{\mu \nu}-4 \frac{L_{F F}}{L_{F}} F^{\mu \alpha} F_{\alpha}^{v}\right) k_{\mu} k_{v}=0 .
$$

which exhibits the fundamental feature of NLED, i.e., the effective metric.

Then, by taking the derivative of the last expression, one arrives to

$$
k^{\nu} \nabla_{v} k_{\alpha}=4\left(\frac{L_{F F}}{L_{F}} F^{\mu \beta} F_{\beta}^{v} k_{\mu} k_{\nu}\right)_{\mid \alpha} .
$$

Eq.(48) shows that the nonlinear Lagrangian introduces a term acting as a force accelerating the photon. This acceleration of any photon which is traversing over weak background electromagnetic fields in a vacuum is the new physical element that we argue hereafter would be responsible for the Pioneer anomaly.

\subsubsection{NLED photon acceleration: What Earth receivers are reading off radio signals from interplanetary spacecraft transponders - The case Pioneer anomaly}

If NLED is to play a significant role at the macroscopic scale, this should occur at the intermediary scales of clusters of galaxies or the interclusters medium, wherein most observations show that the magnetic fields are almost uniform (and of the same order of magnitude ${ }^{8}$ ), unlike the dipolar magnetic fields of the Sun and planets. However, galaxies are gravitationally bound systems, whereas the cosmic expansion is acting at the cluster of galaxies scale. Thus, the magnetic field (B) in clusters of galaxies (IGMF) depends on the cosmic time $\left(B=B_{0} a^{-2}\right)$. So, the $\mathbf{B}$ that is relevant to this study is that of the local cluster

\footnotetext{
${ }^{7}$ Next we show that the "acceleration" of photons predicted by NLED may account for the anomalous blueshift indicated by the Pioneer 10/11, Ulysses and Galileo spacecrafts. This will manifest itself as a new frequency shift for the EM waves, in addition to the Doppler shift (special relativity) and the gravitational and cosmological redshift (general relativity), when both of them apply.

${ }^{8}$ Fujii \& Sasaki (2006)
} 
of galaxies Beck (2000). (As regard to the contribution of the CMB radiation see Riess etal. (2004)). ${ }^{9}$ Recently, Vallée (2002) has speculated that the $2 \mu \mathrm{G}$ magnetic field he has observed within the local supercluster of galaxies in cells of sizes of about $100 \mathrm{kpc}$ may extend all the way down to the Sun. We explore further this idea in the framework of NLED and show that it is capable to provide an explanation of the Pioneer anomaly from first principles.

Relation (40) can be cast in the form

$$
g_{\mu v} k^{\mu} k^{v}=4 \frac{L_{F F}}{L_{F}} b^{2}
$$

where $b^{\mu}=F^{\mu \nu} k_{v}$ and $b^{2}=b^{\mu} b_{\mu}$.

As $E=0$, one can write, after averaging over the angular-dependence Bialynicka-Birula \& Bialynicki-Birula (1970):

$$
b^{2}=-\left.\frac{1}{2}|| \vec{k}\right|^{2} B^{2} c^{2}=-\left.\frac{1}{4}|| \vec{k}\right|^{2} F
$$

with $\|\vec{k}\|=\omega / c=2 \pi v / c$. By inserting this relation in (49) yields

$$
g_{\mu v} k^{\mu} k^{v}=-\frac{\omega^{2}}{c^{2}} F \frac{L_{F F}}{L_{F}} .
$$

Taking the $\left(x^{\alpha}\right)$ derivative of Eq.(51) we obtain

$$
2 g_{\mu \nu} k^{\mu}\left(k^{v}\right)_{\mid \alpha}+k^{\mu} k^{\nu}\left(g_{\mu \nu}\right)_{\mid \alpha}=-\left(\frac{\omega^{2}}{c^{2}} F \frac{L_{F F}}{L_{F}}\right)_{\mid \alpha} .
$$

The cosmological expansion will be represented by $g_{\mu v}=a^{2}(\eta) g_{\mu v}^{(\text {local })}$, with $a$ the scale factor, $\eta$ the conformal time, and $g_{\mu \nu}^{(\text {local })}$ the local metric. So, Eq.(52) yields:

$$
2 g_{\mu \nu} k^{\mu}\left(k^{\nu}\right)_{\mid 0}+2\left(\frac{\dot{a}}{a}\right) g_{\mu \nu} k^{\mu} k^{\nu}=-\left(\frac{\omega^{2}}{c^{2}} F \frac{L_{F F}}{L_{F}}\right)_{\mid 0},
$$

where the dot stands for partial derivative w.r.t. $\eta$. Using Eqs.(51) and (53) we obtain ${ }^{10}$

$$
k_{\mu}\left(k^{\mu}\right)_{\mid 0}=\left(\frac{\dot{a}}{a}\right) \frac{\omega^{2}}{c^{2}} F \frac{L_{F F}}{L_{F}}-\frac{1}{2}\left(\frac{\omega^{2}}{c^{2}} F \frac{L_{F F}}{L_{F}}\right)_{\mid 0} .
$$

Now, $\dot{F}=-4\left(\frac{\dot{a}}{a}\right) F$, by recalling that $B^{2} \propto a^{-4}$. Moreover, from the method of the effective metric, it can be shown that $k^{0}$ does not vary with time in the first order approximation unlike $\|\vec{k}\| .{ }^{11}$ Hence

\footnotetext{
${ }^{9}$ The interclusters magnetic field is in any case by far small $\left(10^{-9} \mathrm{G}\right)$ to add a measurable correction even to the cosmological redshift. As for the contribution of the cosmic microwave background (CMB), not only it is too weak but also, the $\mathrm{CMB}$ is pure radiation $(F=0)$, whereas we are interested in the case of a background magnetic field with no significant electric field counter-part, i.e., $E=0$.

${ }^{10}$ By removing the NLED extra term from Eq.(49), this reduces it to $g_{\mu v}^{(\text {local })} k^{\mu} k^{v}=0$ so that the photons would just see the local background metric.

${ }^{11}$ Given a background metric $g_{\mu v}$, as a result of NLED effects photons follow geodesic paths with respect to the effective metric (or any one conformal to it) $g_{\mu \nu}^{(e f f)}=g_{\mu \nu}-4 \frac{L_{F F}}{L_{F}} F_{\mu}^{\alpha} F_{\alpha v}$ (see Novello et al. (2000),Novello \& Salim (2001)). Thus, following the usual analysis on the gravitational frequency shift
} 


$$
k_{\mu}\left(k^{\mu}\right)_{\mid 0}=-\frac{\omega}{c}\left(\frac{\dot{\omega}}{c}\right) .
$$

By inserting relation (55) in (54), and then expanding and arranging, one finds

$$
\left(\frac{\dot{v}}{v}\right)=-\left(\frac{\dot{a}}{a}\right) \frac{Q+2 F Q_{F}}{1-Q}
$$

where we have set $Q=F \frac{L_{F F}}{L_{F}}$ and $Q_{F}=\partial Q / \partial F$.

At present cosmological time $(t)$, and for a duration very short as compared to the universe age, Eq.(56) reduces to

$$
\left(\begin{array}{c}
\dot{v} \\
v
\end{array}\right) \simeq-H_{0} \frac{Q+2 F Q_{F}}{1-Q}
$$

where ( $\dot{v}$ is the photon frequency $t$-derivative). $\dot{v} \neq 0$ if and only if a) the NLED contribution is non-null, i.e., $L_{\mathrm{FF}} \neq 0$, and b) $F$ depends on time.

\subsubsection{NLED alla NPS as explanation of the Pioneer puzzle}

The explicit form of this general nonlinear Lagrangian (which simulates the effect of dark energy in.Novello et al. (2004)) reads

$$
L=-\frac{1}{4} F+\frac{\gamma}{F}, \quad \text { or } \quad L=-\frac{1}{4} F+\frac{\gamma_{n}}{F^{n}},
$$

where $n$ is a strictly positive integer. From Eqs. $(56,58)$, the time variation of the photon frequency, due to interaction with very weak $\mathbf{B}(t)$ fields, reads

$$
\left(\frac{\dot{v}}{v}\right)=A_{n} \gamma_{n} \frac{4 n \gamma_{n}-(2 n+1) F^{n+1}}{\left(F^{n+1}+4 n \gamma_{n}\right)\left[F^{n+1}+4 n(n+2) \gamma_{n}\right]}
$$

with $A_{n}=4 H_{0} n(n+1)$. Notice that $\gamma_{n}$ should be negative in order to guarantee that the Lagrangian is bound from below (see Landau \& Lifchiftz (1970), sections 27 and 93), $\gamma_{n}=$ $-\left(B_{n} c\right)^{2(n+1)}$. Also, it is worth noticing that Eq.(59) in the nearly-zero field limit $(B \rightarrow 0)$ would reduce to

$$
\left(\frac{\dot{v}}{v}\right)=H_{0}\left(\frac{n+1}{n+2}\right)
$$

which implies a blueshift.

\subsection{Discussion and conclusion}

We stress that the NLED is a universal theory for the electromagnetic field, with $\gamma_{n=1}=\gamma$ in Eq.(58) being a universal constant. The value of $\gamma$ was fixed in Novello et al. (2004) by using

but with $g_{\mu \nu}^{(e f f)}$ replacing $g_{\mu \nu}$, one gets $k^{0} c=\omega_{0} / \sqrt{g_{00}^{(e f f)}}$ (see Landau \& Lifchiftz (1970), section 88), where $v_{0}=\omega_{0} / 2 \pi$ denotes the photon frequency in flat Minkowski spacetime. Thus, discarding the cosmological redshift (subsequent to the time dependence of the curvature), the variation of $k^{0}$ with time can be neglected in the first order approximation, since $F^{0 \alpha} F_{\alpha}^{0}=F_{0}^{\alpha} F_{\alpha 0}=0$ in the case of a zero electric field. 
the CMB constraint. ${ }^{12}$ Indeed, in the standard model of cosmology the CMB is well described by Maxwell theory, which is likely to give a good account of the magnetic fields in galaxies too. Notwithstanding, the processes at the origin of both the seed magnetic field in the interclusters medium and clusters of galaxies are not yet clearly understood. Hence, if NLED is to play a significant role at the macroscopic scale, this should occur at the intermediary scales of clusters of galaxies or the interclusters medium.

Then, considering the possibility that the NLED correction terms described above come into play at these scales, one gets the following ordering of strengths: $B_{\text {Universe }} \ll B_{\text {Intercluster }} \ll$ $B_{1} \lesssim B_{\text {Cluster }} \lesssim B_{\text {Galaxy }}$. Turning back to the Pioneer anomaly, a good accordance is obtained with $B_{\mathrm{LSC}}=1.77 B_{1}$ (case $n=1$ ) Riess etal. (2004). Thus, setting $B_{1}=\frac{1}{c}|\gamma|^{1 / 4}$, one finds $B_{1}=$ $0.008 \pm 0.002 \mu \mathrm{G}$ Riess etal. (2004). ${ }^{13}$

Thence, to compute the effect (drift) on the Pioneer communication signal frequencies (uplink and downlink), we need only to introduce the value of the strength of the local supercluster B-field: $B_{\mathrm{LSC}} \sim 10^{-8}-10^{-7} \mathrm{G}$ Blasi \& Olinto (1999). This involves $Q>0$. But then, since relation (51) implies $Q<1$, and the NLED theory must be such that $L_{\mathrm{F}}<0$ (hence, $F^{n+1}+$ $4 n \gamma_{n}>0$ ) for the energy density of the EM field to be positive definite [see Novello et al. (2000), appendix B], which entails $\mathbf{B}>B_{1}$, one can verify that the equation (59) implies a blueshift. Meanwhile, the impressive accordance of the data from Voyager 1 and 2 magnetometers with Parker's theory (see Fig. 2) constraints $B_{\mathrm{LSC}}$ to be less than $0.022 \mu \mathrm{G}$ within the solar system up to the heliopause. Hence, we may conclude that $0.01 \mu \mathrm{G}<B_{\mathrm{LSC}}<0.022 \mu \mathrm{G}$ within the solar system. By recalling that the uplink frequency of Pioneer 10 and 11 spacecrafts is $v=$ $2.2 \mathrm{GHz}$, one obtains for the median value $B_{\mathrm{LSC}}=0.018 \mu \mathrm{G}$ (both expressions are normalized by $\left.\left[\frac{\mathrm{H}_{0}}{70 \mathrm{~km} \mathrm{~s}^{-1} \mathrm{Mpc}^{-1}}\right]\right)$. Then Eq.(60) renders

$$
\left(\frac{\dot{v}}{v}\right)=2.8 \times 10^{-18} \mathrm{~s}^{-1}, \quad \text { or equivalently } \quad \frac{d \Delta v}{d t}=6 \times 10^{-9} \frac{\mathrm{Hz}}{\mathrm{s}},
$$

with $\Delta v$ being the frequency drift pointed out earlier.

\section{A digression on interplanetary magnetic field and NLED effects:}

It has been pointed out that the strength of the IPMF could severly minimize the NLED effects because it will overrun the interstellar or intergalactic magnetic fields at heliocentric distances. Notwithstanding, the actual data from Voyager $1 / 2$ spacecrafts of the IPMF average strength (see Fig. 2) are both consistent with a non-zero local supercluster magnetic field (LSCMF) amounting up to $0.022 \mu \mathrm{G}$ NASA Voyager 1 (1978); NASA Voyager 2 (1978) (the accuracy of the measurements performed by Pioneer $10 / 11$ magnetometers is at best $0.15 \mu \mathrm{G}$, and

\footnotetext{
${ }^{12}$ Nonetheless, we stress that a conclusive method of fixing $\gamma$ should benefit of a dedicated laboratory experiment, in the same spirit that it was done, for instance, to fix the electron charge through Millikan's experiment.

${ }^{13}$ A clean estimate of $B_{1}$ from our definition of $\gamma_{n} \equiv-\left(B_{n} c\right)^{2(n+1)}$, below Eq.(59), and the one in Novello et al. (2004): $\gamma \equiv-\hbar^{2} \mu^{8}$. On account that $a_{c}=\left(1+z_{c}\right)^{-1}$ and $\gamma=-\hbar^{2} \mu^{8}=-\left(B_{1} c\right)^{4}$, Eq.(13) of Novello et al. (2004) rewrites $B_{1}^{4} / \mu_{0} B_{0}^{2}=1.40 \rho_{c} c^{2}$ (A1). Thus $a_{c}=\left(B_{0}^{4} c^{4} / 3 \hbar^{2} \mu^{8}\right)^{1 / 8}$ yields $B_{1}=3^{-1 / 4}\left(1+z_{c}\right)^{2} B_{0}(\mathrm{~A} 2)$. Then, combining both relations (A1) and (A2) one gets : $B_{0}=0.02\left(1+z_{c}\right)^{-4} \mu \mathrm{G}(\mathrm{A} 3), B_{1}=0.016(1+$ $\left.z_{c}\right)^{-2} \mu \mathrm{G}(\mathrm{A} 4)$. Now, Riess etal. found evidence for a transition from cosmic deceleration to acceleration at redshift $z_{c}=0.46 \pm 0.13$ [A. G. Riess etal., ApJ 607, 665 (2004)]. Inserting the latter figures in relations (A3) and (A4) yields : $B_{0}=(0.005 \pm 0.002) \mu \mathrm{G}$ (A5), $B_{1}=(0.008 \pm 0.002) \mu \mathrm{G}(\mathrm{A} 6)$. Since CMB is pure radiation (i. e., $E=B c$ not equal to zero on average), we consider that relations (A4) and (A6) give a better estimate of $B_{1}$ than the one put forward in Novello et al. (2004).
} 
$0.022 \mu \mathrm{G}$ for the low field system of Voyager 1/2 magnetometers Solar Wind (1980)). Besides, it is just beyond the Saturn orbit, $\sim 10$ Astronomical Units (AU), that the anomaly begins to be clearly observed. Surprisingly, it is just after passing the Saturn orbit that the strength of the magnetic field vehiculated by the solar wind gets down the strength associated to the insterstellar and intergalactic magnetic fields, as one can verify by perusing on Fig. 2 (see Refs.NASA Voyager 1 (1978); NASA Voyager 2 (1978)). Thus, since a magnetic field cannot shield (or block) any another magnetic field (the stronger field can only reroute the weaker field, otherwise it would violate Maxwell's laws), then it follows that the LSCMF has its magnetic influence extended upto nearly the location of the Saturn orbit, and in this way it forces the photons being emitted by the Pioneer spacecrafts from larger heliocentric distances to get accelerated due to the NLED effects. Besides, notice that Mitra et al. (2003) also shows that the local cloud of interstellar gas in HII regions does not keep out the Galactic magnetic field.

"En passant", we call to the reader's attention the fact that some workers in the field have claimed that the effect should have shown up already at the small distance corresponding to Mars, Jupiter or Saturn orbits, because of the high technology involved in the tracking of planet orbiting spacecrafts as Galileo and Cassini or the Mars' nonroving landers, which would allow to single out the anomaly at those heliocentric distances. However, as those spacecrafts are inside the region where the solar wind dominates, this definitely precludes the NLED photon acceleration effect to show up at those distances since the much higher magnetic field there would introduce a negligible NLED effect, and as stated below the solar pressure influence on the signal frequency is still large.

On the other hand, although one can use the time of flight of photons during tracking of planets with orbiting spacecrafts (by combining range and Doppler data over a spacecraft orbit) to tightly determine the range from Earth to that given planet's center of mass, the impediment to single out the radio-signal frequency shift remains the same pictured above: from one side the strength of both the host planet magnetic field and the solar magnetic field at those distances are still large, what blocks the nonlinear action of the LSCMF, and from the large up to $20 \mathrm{AU}$ so as to allow the show up of the NLED frequency shift which is much smaller. Moreover, within a heliocentric distance $\sim 100$ AU the IPMF keeps stronger. Thus, it reduces for all practical purposes the IPMF contribution to the effects of NLED (see further arguments from our direct estimate of $B_{1}$ in Footnote 13 above, and in Riess etal. (2004)), leaving room for the sole contribution of the residual IGMF in the solar system.

Finally, the new frequency shift that is predicted by NLED is not seen yet in the laboratory because of some of the following reasons: a) the most important, the strength of the Earth magnetic field is much larger than the one required in the NLED explanation of the anomaly for the effect to show up, and b) the coherence time $\tau=1 / \Delta v$ of EM waves in present atomic clocks (frequency width $\Delta v>0.01 \mathrm{~Hz}$, or otherwise stated $c \tau<0.2 \mathrm{AU}$ ) is too short as compared to the time of flight of photons from Pioneer 10/11 spacecrafts past 20 AU. Nonetheless, if the conditions demanded by our model were satisfied this effect will certainly be disentangled in a dedicated experiment where, for instance, the Earth magnetic field is kept outside the case containing an experimental set up where a very weak magnetic field is maintained inside, a source of photons set to travel and a receiver data-collecting.

\section{Acknowledgements}

The author would like to sincerely thank Jean-Paul Mbelek (Service d'Astrophysique, C. E. Saclay, 91191 Gif-sur-Yvette Cedex, France), Mário Novello (Instituto de Cosmologia, 


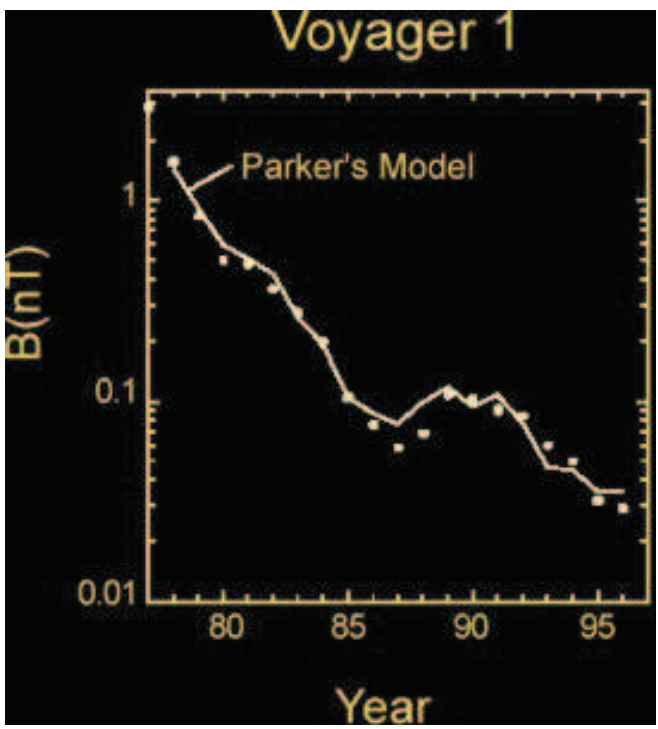

(a) Subfigure 1

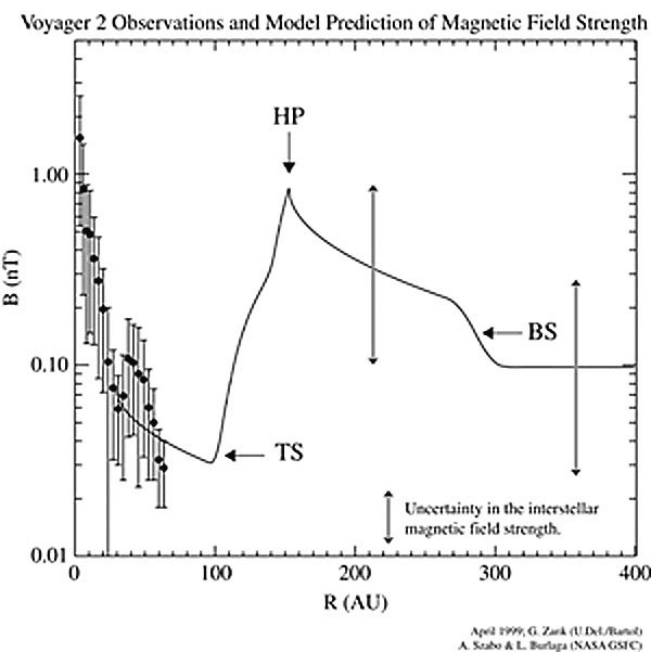

(b) Subfigure 2

Fig. 2. Data from Voyager $1 / 2$ spacecrafts of the interplanetary magnetic field (IPMF) average strength. Subfigure 1 presents measurements by Voyager 1 of the strength of the IPMF [nT] as a function of time [Yr]. The continuous line represents the predictions of Parker's model, and the dots the Voyager 1 data. Subfigure 2 shows the dependence with the distance [AU] of the IPMF [nT], as detected by Voyager 2, against the theoretical prediction. Credit: http://spacephysics.ucr.edu/images/swq2-04.jpg, and http://interstellar.jpl.nasa.gov/interstellar/probe/interstellar/images/ 07BupstrmSuess-25lg.gif 
Relatividade e Astrofísica (ICRA-BR), Centro Brasileiro de Pesquisas Físicas (CBPF)), and José Martins Salim (Instituto de Cosmologia, Relatividade e Astrofísica (ICRA-BR), Centro Brasileiro de Pesquisas Físicas (CBPF)) for the former collaboration in this direction of research. Also Prof. Remo Ruffini, Director General of the International Center for Relativistic Astrophysics Network (ICRANet), and the International Coordinating Centre, Pescara, Italy are thanked for the hospitality given during the final preparation of this work (September 2010).

\section{References}

Abramovici, A.. \& Vager, Z. (1986). Phys. Rev. D 34, 3240

Anderson, J. D., et al. (1995). Astrophys. J. 448, 885

Anderson, J. D. , et al. (1998). Phys. Rev. Lett. 81, 2858

Anderson, J. D. , et al.. (2002). Phys. Rev. D 65, 082004

Beck, R. (2001). Astrophys. Sp. Sc. Rev. 99, 243, in Proceedings of The Astrophysics of Galactic Cosmic Rays Conference, eds. R. Diehl etal., Kluwer, Dordrecht

Bialynicka-Birula, Z. Bialynicki-Birula, I. (1970). Phys. Rev. D 2, 2341

Blasi, P. \& Olinto, A. V. (1999). Phys. Rev. D 59, 023001

Burke, D. L., et al. (1997). Phys. Rev. Lett. 79, 1626

Born, M. \& Infeld, L. (1934). Proc. Roy. Soc. Lond. A 144, 425

De Lorenci, V. A.., Klippert, R., Novello, M. \& Salim, M., (2002). Phys. Lett. B 482, 134

Delphenich, D. H. (2003). Nonlinear electrodynamics and QED. arXiv: hep-th/0309108

Delphenich, D. H. (2006). Nonlinear optical analogies in quantum electrodynamics. arXiv: hep-th/0610088

Denisov, V. I., Denisova, I. P. \& Svertilov, S. I. (2001a). Doklady Physics, Vol. 46, 705.

Denisov, V. I., Denisova, I. P., Svertilov, S. I. (2001b). Dokl. Akad. Nauk Serv. Fiz. 380, 435

Denisov, V. I., Svertilov, S. I. (2003). Astron. Astrophys. 399, L39

Dittrich, W. \& Gies, H. (1998). Phys Rev.D 58, 025004

Dolag, K., Bartelmann, M. \& Lesch, H. (1999). Astron. Astrophys. 348, 351

Einstein, A.. (1936). Lens-Like Action of a Star by the Deviation of Light in the Gravitational Field. Science, New Series, Vol. 84, No. 2188, 506-507

Einstein, A.. (1911). On The influence of gravitation on the propagation of light. Annalen Phys. 35, 898-908

Einstein, A.. (1912). The Speed of Light and the Statics of the Gravitational Field. Annalen Phys. 38, 355-359

Einstein, A.. (1912). On the Theory of the Static Gravitational Field. Annalen Phys.38, 443-458

Fujii, Y. \& Sasaki, M. (2006). report astro-ph/0608508

The value of the magnetic field measured in the Galaxy is $B_{\text {Galaxy }}=6 \pm 2 \times 10^{-6} \mathrm{G}$ Beck (2000) and that of the local cluster of galaxies is $B_{\text {Cluster }} \sim 2-3 \times 10^{-6} \mathrm{G}$ Beck (2000), and for extragalactic clusters as Abell 400, Hydra A measurements provide $B_{\mathrm{EG}-\mathrm{C}} \sim$ $3-20 \times 10^{-6} \mathrm{G}$ Vogt \& Ensslin (2003).

Garcia, A.. \& Plebanski, J. (1989). J. Math. Phys. 30, 2689

Hadamard, J. (1903) Leçons sur la propagation des ondes et les equations de l'Hydrodynamique (Hermann, Paris, 1903)

Following Hadamard Hadamard (1903), the surface of discontinuity of the EM field is denoted by $\Sigma$. The field is continuous when crossing $\Sigma$, while its first derivative presents a finite discontinuity. These properties are specified as follows: $\left[F_{\mu \nu}\right]_{\Sigma}=0$, 
$\left[F_{\mu v \mid \lambda}\right]_{\Sigma}=f_{\mu \nu} k_{\lambda}$, where the symbol $\left[F_{\mu \nu}\right]_{\Sigma}=\lim _{\delta \rightarrow 0^{+}}\left(\left.J\right|_{\Sigma+\delta}-\left.J\right|_{\Sigma-\delta}\right)$ represents the discontinuity of the arbitrary function $J$ through the surface $\Sigma$. The tensor $f_{\mu \nu}$ is called the discontinuity of the field, $k_{\lambda}=\partial_{\lambda} \Sigma$ is the propagation vector, and symbols " " and "॥" stand for partial and covariant derivatives.

Heisenberg, W. \& Euler, H., (1936). Zeit. Phys. 98, 714

Iorio, L. (2006a). e-print gr-qc/0601055.

Iorio, L. (2006b). e-print gr-qc/0608068.

Iorio, L. (2006c). e-print gr-qc/0608101

Kandus, A.., Kerstin, K. E. \& Tsagas, C. (2010). Primordial magneto-genesis. arXiv: 1007.3891 [astro-ph.CO]

Kunze, K. E. (2008). Phys. Rev. D 77, 023530. arXiv:0710.2435 [astro-ph]

Landau, L. D. \& Lifchiftz, E. (1970). Théorie des Champs. (Editions MIR, Moscou)

Lichnerowicz, A., (1962). Elements of Tensor Calculus, (John Wiley and Sons, New York). See also Relativistic Hydrodynamics and Magnetohydrodynamics (W. A. Benjamin, 1967), and Magnetohydrodynamics: Waves and Shock Waves in Curved Space-Time (Kluwer, Springer, 1994)

Lundstrom, E., et al. (2006). Phys. Rev. Lett. 96: 083602

Lundin, J., Brodin, G., \& Marklund, M. (2006). PHYSICS OF PLASMAS 13: 102102. e-Print Archive: hep-ph/0606136; V. A. De Lorenci et al., Phys. Lett. B 482: 134-140 (2000);

Marklund, M. \& Shukla, P. K. (2006). Rev. Mod. Phys. 78: 591-640

Markwardt, C. B. (2002). preprint gr-qc/0208046.

Mbelek, J. P. \& Michalski, M. (2004). Int. J. Mod. Phys. D 13, 865

Mbelek, J. P. (2005). Int. J. Mod. Phys. A 20, 2304

Mbelek, J. P., Mosquera Cuesta, H. J., Novello, M. \& Salim, J. M. (2007). Nonlinear electrodynamics and the Pioneer 10/11 spacecraft anomaly. Europhys. Lett. 77, 19001

Mendonça, J. T., (2000). Theory of photon acceleration. (Taylor \& Francis 2000). Print ISBN: 978-0-7503-0711-6. eBook ISBN: 978-1-4200-3327-4 See also Mendonca et al. (2006). e-Print Archive: hep-ph/0607195

Milgrom, M. (2001). Acta Phys. Polon. B 32, 3613.

Milgrom, M. (2002). New Astron. Rev. 46, 741

Mitra, D., et al. (2003). Astron. Astrophys. 398, 993

Mosquera Cuesta, H. J., Salim, J. M., (2004). Mon. Not. Roy. Astron. Soc. 354, L55-L59

Mosquera Cuesta, H. J., Salim, J. M. (2004). Astrophys. J. 608, 925. e-print astro-ph/0307519 (2003).

Mosquera Cuesta, H. J., de Freitas Pacheco, J. A.., \& Salim, J. M. (2006). Int. J. Mod. Phys. A 21: 43-55

Mosquera Cuesta, H. J. \& Lambiase, G. (2009). Phys. Rev. D 80:023013. e-Print: arXiv:0907.3678 [astro-ph.CO]

Mosquera Cuesta, H. J. (2010). L'energie sombre: Un mirage cosmique? Luminosity distance $\neq$ proper distance: A cosmological dissimilitude induced by nonlinear electrodynamics. INVISIBLE UNIVERSE: Proceedings of the Conference. AIP Conference Proceedings, Volume 1241, 1083-1092

Novello, M., De Lorenci, V. A., Salim, J. M., and Klippert, R. (2000). Phys. Rev. D 61, 045001

Novello, M., Salim, J. M., Phys. Rev. D 63, 083511 (2001)

Novello, M., Pérez Bergliaffa, S. E. \& Salim, J. M. (2004). Phys. Rev. D 69, 127301

Pagels, H.\& Tomboulis, E. (1978). Nuc. Phys. B 143, issue 3, 485

Pérez Martínez, A., Pérez Rojas, H., \& Mosquera Cuesta, H. J. (2003). Eur. Phys. Journ. C, 29, 


\section{1}

Plebanski, J. (1970). Lectures on nonlinear electrodynamics. (Nordita, Copenhagen, 1970)

Pioneer Explorer Collaboration. (2005). e-print gr-qc/0506139

Quevedo, H. B. (2005). e-print gr-qc/0501006

Rañada, A. F. (2003). Europhys. Lett. 63, 653

Rañada, A. F. (2005). Found. Phys. 34, 1955

Riess, A.. G., etal. (2004). ApJ 607, 665

Schwinger, J. (1951). Phys. Rev. 82, 664

Solar Wind: Detection Methods and Long-term Fluctuations, at http://herkules . oulu. fi/isbn9514271955/isbn9514271955.pdf

Stephani, H. (2004). Relativity. Cambridge University Press, Cambridge, UK, Third Edition, pp 44

Tangen, K. (2006). Could the Pioneer anomaly have a gravitational origin? gr-qc/0602089:

Tolman, R. C. (1934). Effect of Inhomogeneity on Cosmological Models. Proc. Nat. Ac. Sc. 20, 169. See also Tolman, R. C. \& Ehrenfest, P. (1930). Temperature Equilibrium in a Static Gravitational Field. Phys. Rev. 36, 1791

Turyshev, S. G. \& Toth, V. T. (2010). Living Rev. Rel. 13, 4

Vallée, J. P. (2002). Astronom. J., 124, 1322

Vogt, C. \& Ensslin, T. A.. (2003). Astron. Astrophys. 412, 373.

Voyager 1 Observations and Parker's Model of Interplanetary Magnetic Field (IPMF) Strength, http://spacephysics.ucr.edu/images/swq2-04.jpg, http:// www.phy6.org/Education/wtermin.html

Voyager 2 Observations and Model Prediction of IPMF Strength, at http:// interstellar.jpl.nasa.gov/interstellar/probe/interstellar/ images/07BupstrmSuess-25lg.gif

Whitmire, D. P. \& Matese, J. J. (2003). Icarus 165, 219

\section{APPENDIX: The method of effective geometry}

Following Hadamard (1903), the surface of discontinuity ${ }^{14}$ of the EM field is denoted by $\Sigma$. The field is continuous when crossing $\Sigma$, while its first derivative presents a finite discontinuity. These properties are specified as follows

$$
\begin{aligned}
{\left[B^{\mu}\right]_{\Sigma} } & =0, \quad\left[\partial_{\alpha} B^{\mu}\right]_{\Sigma}=b^{\mu} k_{\alpha}, \quad\left[\partial_{\alpha} E^{\mu}\right]_{\Sigma}=e^{\mu} k_{\alpha} \\
{\left[F_{\mu \nu}\right]_{\Sigma} } & =0, \quad\left[F_{\mu \nu \mid \lambda}\right]_{\Sigma}=f_{\mu \nu} k_{\lambda},
\end{aligned}
$$

where the symbol

$$
\left[F_{\mu \nu}\right]_{\Sigma} \equiv \lim _{\delta \rightarrow 0^{+}}\left(\left.J\right|_{\Sigma+\delta}-\left.J\right|_{\Sigma-\delta}\right)
$$

represents the discontinuity of the arbitrary function $J$ through the surface $\Sigma$. The tensor $f_{\mu \nu}$ is called the discontinuity of the field, and $k_{\lambda}=\partial_{\lambda} \Sigma$ is the propagation vector. In Eq. (62), the symbol "|" stands for partial derivative;

\footnotetext{
${ }^{14}$ Of course, the entire discussion onwards could alternatively be rephrased in terms of concepts more familiar to the astronomy community as that of light rays used for describing the propagation of electromagnetic waves in the geometric optics approximation.
} 
Here-after we want to investigate the effects of nonlinearities of very strong magnetic fields in the evolution of electromagnetic waves described onwards as the surface of discontinuity of the electromagnetic field (represented here-to-fore by $F_{\mu v}$ ). For this reason we will restrict our analisys to the simple class of gauge invariant Lagrangians defined by $L=L(F)$. From the least action principle we obtain the following field equation

$$
\left(L_{F} F^{\mu v}\right)_{\| \mu}=0 \text {. }
$$

Applying the Hadamard conditions (62) and (63) to the discontinuity of the field in Eq.(64) we obtain

$$
L_{F} f^{\mu v} k_{v}+2 L_{F F} \xi^{\mu v} k_{v}=0,
$$

where $\xi$ is defined by $\xi \doteq F^{\alpha \delta} f_{\alpha \delta}$. Both, the discontinuity conditions and the electromagnetic field tensor cyclic identity lead to the following dynamical relation

$$
f_{\mu \nu} k_{\lambda}+f_{v \lambda} k_{\mu}+f_{\lambda \mu} k_{v}=0
$$

In the particular case of a polarization such that $\xi=0$, it follows from Eq.(64) that $f^{\mu v} k_{v}=0$. Thus using this result, and multiplying Eq.(66) by $k^{\lambda}$ we obtain

$$
f_{\mu v} k^{\mu} k^{v}=0 .
$$

This equation states that for this particular polarization the discontinuity propagates with the metric $f_{\mu \nu}$ of the background space-time. For the general case, when $\xi \neq 0$, we multiply Eq.(66) by $k_{\alpha} g^{\alpha \lambda} F^{\mu v}$ to obtain

$$
\xi k_{v} k_{\mu} g^{\mu v}+2 F^{\mu v} f_{v}^{\lambda} k_{\lambda} k_{\mu}=0 .
$$

From this relation and Eq.(65) we obtain the propagation law for the field discontinuities, in this case given as

$$
\left(L_{F} g^{\mu v}-4 L_{F F} F_{\alpha}^{\mu} F^{\alpha v}\right) k_{\mu} k_{v}=0
$$

where $F^{\mu}{ }_{\alpha} F^{\alpha v}=-B^{2} h^{\mu v}-B^{\mu} B^{v}$. Eq.(69) allows to interpret the term inside the parenthesis multiplying $k^{\mu} k^{v}$ as an effective geometry

$$
g_{\text {eff }}^{\mu v}=L_{F} g^{\mu v}-4 L_{F F} F_{\alpha}^{\mu} F^{\alpha v} .
$$

Hence, one concludes that the discontinuities will follow geodesics in this effective metric.

\section{APPENDIX-1: NPS theory applied to cosmology}

To discuss the evolution of a universe model driven by the NPS NLED, the electromagnetic ( EM) field described by Eq.(34) can be taken as source in Einstein equations to obtain a toy model for the evolution of the universe which displays accelerate expansion. Such phase of acceleration runs into action when the nonlinear EM term takes over the term describing other matter fields. This NLED theory yields ordinary radiation plus a dark energy component with $w<-1$ (phantom-like dynamics). Introducing the notation ${ }^{15}$, the EM field can act as

\footnotetext{
${ }^{15}$ Due to the isotropy of the spatial sections of the Friedman-Robertson-Walker (FRW) model, an average procedure is needed if electromagnetic fields are to act as a source of gravity Tolman (1934).
} 
a source for the FRW model if $\left\langle E_{i}\right\rangle_{\left.\right|_{V}}=0,\left\langle B_{i}\right\rangle_{\left.\right|_{V}}=0,\left\langle E_{i} B_{j}\right\rangle_{\left.\right|_{V}}=0,\left\langle E_{i} E_{j}\right\rangle_{\left.\right|_{V}}=-\frac{1}{3} E^{2} g_{i j}$, and $\left\langle B_{i} B_{j}\right\rangle_{V_{V}}=-\frac{1}{3} B^{2} g_{i j} .{ }^{16}$ When these conditions are fulfilled, a general nonlinear Lagrangian $L(F)$ yields the energy-momentum tensor $\left(L_{F}=d L / d F, L_{F F}=d^{2} L / d F^{2}\right)^{17}$

$$
\begin{gathered}
\left\langle T_{\mu v}\right\rangle_{\left.\right|_{V}}=(\rho+p) v_{\mu} v_{v}-p g_{\mu v} \\
\rho=-L-4 E^{2} L_{F}, \quad p=L+\frac{4}{3}\left(E^{2}-2 B^{2}\right) L_{F},
\end{gathered}
$$

Hence, when there is only a magnetic field, the fluid can be thought of as composed of a collection of non-interacting fluids indexed by $k$, each of which obeys the equation of state $p_{k}=\left(\frac{4 k}{3}-1\right) \rho_{k}$, composed of ordinary radiation with $p_{1}=\frac{1}{3} \rho_{1}$ and of another fluid with equation of state $p_{2}=-\frac{7}{3} \rho_{2}$. It is precisely this component with negative pressure that may drive accelerate expansion through Friedmann equations, as was shown in Novello et al. (2004).

Thus a volumetric spatial average of a quantity $X$ at the time $t$ by $\langle X\rangle_{\left.\right|_{V}} \equiv \lim _{V \rightarrow V_{0}} \frac{1}{V} \int X \sqrt{-g} d^{3} x$, where $V=\int \sqrt{-g} d^{3} x$, and $V_{0}$ is a sufficiently large time-dependent three-volume. (Here the metric sign convention (+- - ) applies).

${ }^{16}$ Let us remark that since we are assuming that $\left\langle B_{i}\right\rangle_{V_{V}}=0$, the background magnetic fields induce no directional effects in the sky, in accordance with the symmetries of the standard cosmological model.

${ }^{17}$ Under the same assumptions, the EM field associate to Maxwell Lagrangian generates the stress-energy tensor defined by Eq.(71) but now $\rho=3 p=\frac{1}{2}\left(E^{2}+B^{2}\right)$. 


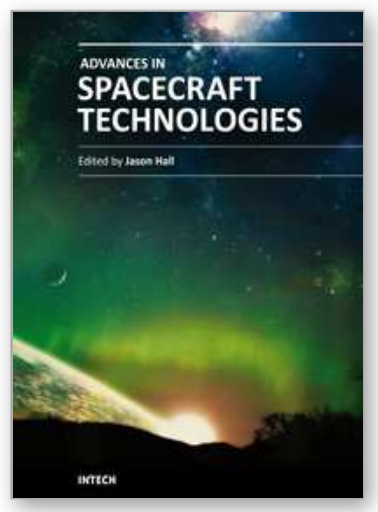

\author{
Advances in Spacecraft Technologies \\ Edited by Dr Jason Hall
}

ISBN 978-953-307-551-8

Hard cover, 596 pages

Publisher InTech

Published online 14, February, 2011

Published in print edition February, 2011

The development and launch of the first artificial satellite Sputnik more than five decades ago propelled both the scientific and engineering communities to new heights as they worked together to develop novel solutions to the challenges of spacecraft system design. This symbiotic relationship has brought significant technological advances that have enabled the design of systems that can withstand the rigors of space while providing valuable space-based services. With its 26 chapters divided into three sections, this book brings together critical contributions from renowned international researchers to provide an outstanding survey of recent advances in spacecraft technologies. The first section includes nine chapters that focus on innovative hardware technologies while the next section is comprised of seven chapters that center on cutting-edge state estimation techniques. The final section contains eleven chapters that present a series of novel control methods for spacecraft orbit and attitude control.

\title{
How to reference
}

In order to correctly reference this scholarly work, feel free to copy and paste the following:

Herman J. Mosquera Cuesta (2011). Nonlinear Electrodynamics: Alternative Field Theory for Featuring Photon Propagation Over Weak Background Electromagnetic Fields and what Earth Receivers Read off Radio Signals from Interplanetary Spacecraft Transponders, Advances in Spacecraft Technologies, Dr Jason Hall (Ed.), ISBN: 978-953-307-551-8, InTech, Available from: http://www.intechopen.com/books/advances-in-spacecrafttechnologies/nonlinear-electrodynamics-alternative-field-theory-for-featuring-photon-propagation-over-weakbackgr

\section{INTECH}

open science | open minds

\section{InTech Europe}

University Campus STeP Ri

Slavka Krautzeka 83/A

51000 Rijeka, Croatia

Phone: +385 (51) 770447

Fax: +385 (51) 686166

www.intechopen.com

\section{InTech China}

Unit 405, Office Block, Hotel Equatorial Shanghai

No.65, Yan An Road (West), Shanghai, 200040, China 中国上海市延安西路65号上海国际贵都大饭店办公楼405单元

Phone: +86-21-62489820

Fax: +86-21-62489821 
(C) 2011 The Author(s). Licensee IntechOpen. This chapter is distributed under the terms of the Creative Commons Attribution-NonCommercialShareAlike-3.0 License, which permits use, distribution and reproduction for non-commercial purposes, provided the original is properly cited and derivative works building on this content are distributed under the same license. 